\title{
Rationality of escalating commitment in information systems project management: An inter-disciplinary perspective
}

\author{
Dmitriy V. Chulkov \\ (School of Business, Indiana University Kokomo, Kokomo Indiana 46904-9003, USA)
}

\begin{abstract}
Escalation of commitment has been linked to losses in information systems (IS) projects. Understanding the nature and the rationality of escalation allows the firm to promote optimal project management practices. This study takes an inter-disciplinary approach and draws on research from economics and management to create a model of irrational escalation and a model of rational escalation. The forces that contribute to irrational escalation include the responsibility of the same manager for both the project selection and project continuation decisions that create proneness to self-justification, the potential for negative framing of decision options due to large sunk costs, the proximity of project completion and the presence of organizational inertia. Identifying these irrational escalation factors helps design appropriate de-escalation techniques. The rational escalation model draws on the real option theory and the bandit process theory to identify conditions when project continuation is justified by the value of information and the value of flexibility that the firm receives from continuing the project.
\end{abstract}

Key words: project management; project termination; escalation of commitment; management information systems; incentives

\section{Introduction}

Escalation of commitment involves continuation of a project after the decision maker receives negative feedback about the project's performance (Brockner, 1992). Such escalation has been linked to significant losses due to project failures and delays in information systems (IS) and information technology (IT) projects. Several studies (Abdel-Hamid, 1988; Zmud, 1980; Na, et al., 2004) suggest that IS projects are particularly prone to escalation. A number of case studies presented the specifics of the losses that may be associated with escalated projects. For instance, Monteleagre and Keil (2000) discuss the development of an automated baggage handling system for the Denver International Airport. This project faced numerous instances of negative feedback, and was completed 16 months behind schedule and \$2 billion over budget. Keil, et al (2000) report that between thirty and forty percent of IS projects were affected by escalation.

Escalation research in management and economics has identified a number of factors that contribute to the escalation process. One issue that has not been resolved is the distinction between rational and irrational determinants of escalation. Drawing on the literature, this inter-disciplinary study presents a model of irrational escalation and a model of rational escalation. The models identify the factors that contribute, respectively, to the irrational and the rational escalation decisions.

Dmitriy V. Chulkov, Ph.D., associate professor of economics and MIS, School of Business, Indiana University Kokomo; research field: management information systems. 
Understanding that escalation decisions may be the outcome of both irrational and rational decision processes has important implications for preventing escalation in IS project management. Irrational escalation reduces the value of the firm, and various de-escalation studies recommended such measures as a change in the management of the project and improved governance mechanisms including monitoring and regular evaluations of the project's progress. Among others, Kim and Park (2007) note that an important determinant of de-escalation is the presence of control mechanisms such as the Capability Maturity Model (CMM). These monitoring and control mechanisms should be implemented if the determinants of irrational escalation are present in the firm's decision processes. On the other hand, aggressive application of de-escalation techniques under conditions of uncertainty may lead to the cancellation of projects that may ultimately turn out to be successful.

The notion of premature termination of IS projects was discussed by Drummond (2005). Such termination is not in the best interest of the firm. The risk of premature termination exists when the evaluation of the project does not include the full value of the project. The decision to escalate a project may be rational for the firm when it is guided by project evaluation techniques that include such factors as the real option value into the decision making process. Distinguishing rational and irrational escalation decisions in IS project management allows to reduce the risk of premature termination of projects and helps establish optimal project management processes at the firm.

In general, the managerial literature approaches escalation as an irrational phenomenon driven by psychological and organizational factors such as self-justification - the managers' need to appear rational in decisions to themselves and to others (Staw, 1976). In contrast, the economic literature approaches escalation by exploring the rational incentives for project continuation after negative feedback. In agency theory, for instance, the manager in charge of the project may receive information about the project performance before the principal of the firm. This information asymmetry provides the incentive to conceal information and continue the project if the termination of the project negatively impacts the reputation of the manager (Kanodia, et al., 1989). Such escalation is rational for the manager (agent), but irrational for the firm (principal). Other economic theories, such as the real option theory, provide the rationale for escalation decisions that are rational for both the manager and the firm. Fig. 1 summarizes the cases of escalation based on the rationality of the escalation decision.

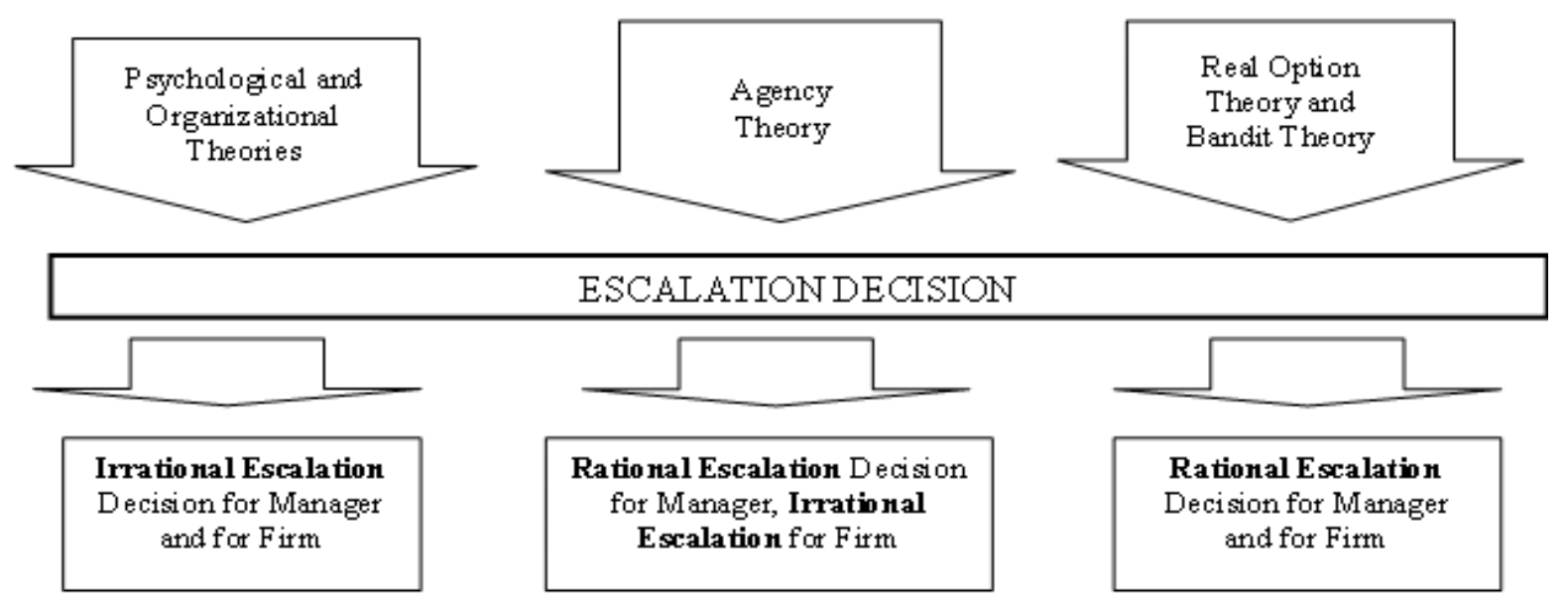

Fig. 1 Rationality of escalation

The objective of this study is to summarize the determinants of escalation in two separate models - the 
irrational escalation model and the rational escalation model. The former includes the determinants of escalation that is irrational for both the manager and the firm. The latter focuses on the rational reasons to continue the project after negative feedback.

\section{Irrational escalation}

Escalation is irrational when it is truly a mistake for both the manager and the firm. Psychological and organizational theories provide insights on the determinants of irrational escalation decisions.

\subsection{Determinants of irrational escalation}

Beginning from the early demonstration of escalation by Staw (1976), self-justification has become the primary explanation for the escalation of commitment. According to the self-justification theory, decision makers are motivated to rectify past losses and attempt to rationalize their actions, or psychologically defend themselves against an apparent error in judgment. Internal self-justification causes an individual desire "to restore consistency between the consequences of his actions and a self-concept of rational decision-making” (Staw, 1976), while external self-justification involves the need of a decision maker to appear rational to other stakeholders such as supervisors.

Norms for consistency in organizations is a theory that complements self-justification in explaining irrational escalation decisions. Staw (1981) presents evidence that consistent administrators are viewed as better leaders. This perception may be acquired through socialization in government and business roles, and contributes to the proneness of managers to escalate their commitment to a project.

Another factor that contributes to irrational project continuation decisions is the size of sunk investment in the project. The sunk cost effect is defined as the propensity of managers to continue a project when a large amount of money has already been committed. A major explanation for the sunk cost effect is the prospect theory. Under the prospect theory (Kahnemann \& Tversky, 1979), individuals exhibit risk-averse behavior when the decision is framed positively and risk-seeking behavior when the decision is framed negatively. It implies that the negative framing of decision as a choice between losses may contribute to irrational continuation of investment by a risk-averse or risk-neutral manager. As withdrawal from a course of action may lead to the sunk costs being viewed as a certain loss, the decision makers become more reluctant to withdraw as sunk costs increase. Experimental evidence (Garland, et al., 1990) suggests that strong negative feedback and professional experience may reduce the sunk cost effect.

An alternative view is provided by the approach-avoidance theory, as escalation may be presented as an instance of the approach-avoidance conflict (Rubin \& Brockner, 1975). In this view, escalation behavior results when driving forces that encourage persistence prevail over restraining forces that encourage abandonment (Brockner \& Rubin, 1985). These competing forces create a conflict over the decision to escalate or terminate a project. The forces that encourage persistence include the size of the payoff for achieving project goals, the cost of withdrawal and the proximity to the goal.

One more determinant of escalation that exists at the organizational level is organizational inertia (Brockner, 1992). With organizational inertia, the need to stop an escalating project may be recognized, but the organization is incapable of quick action. Such inability to react to feedback about the project may affect project continuation 
decisions, especially late in the project life cycle.

\subsection{Irrational escalation model}

The irrational escalation decision is a decision error. Such a decision conflicts with the optimal project management techniques and may be affected by the six determinants of escalation identified in the literature. These determinants include the psychological theories of self-justification, approach-avoidance conflict and the prospect theory, as well as the closely related sunk-cost effect. The social and organizational determinants of irrational escalation include norms for consistency and organizational inertia. Fig. 2 presents the irrational escalation model.

Several implications for the management of IS projects follow from the irrational escalation model. Identifying the determinants that contribute to irrational escalation is helpful for mitigating the problem of IS project waste. De-escalation techniques should be applied for IS projects that exhibit the presence of escalation determinants identified in Fig. 1, including the personal responsibility of managers for both the project selection and project continuation decisions, the negative framing of project termination options due to large sunk costs, organizational size and structure and the proximity of project completion goal. Applying de-escalation techniques may lead to improvement in project development success rate and the reduction of waste due to escalation errors in these cases.

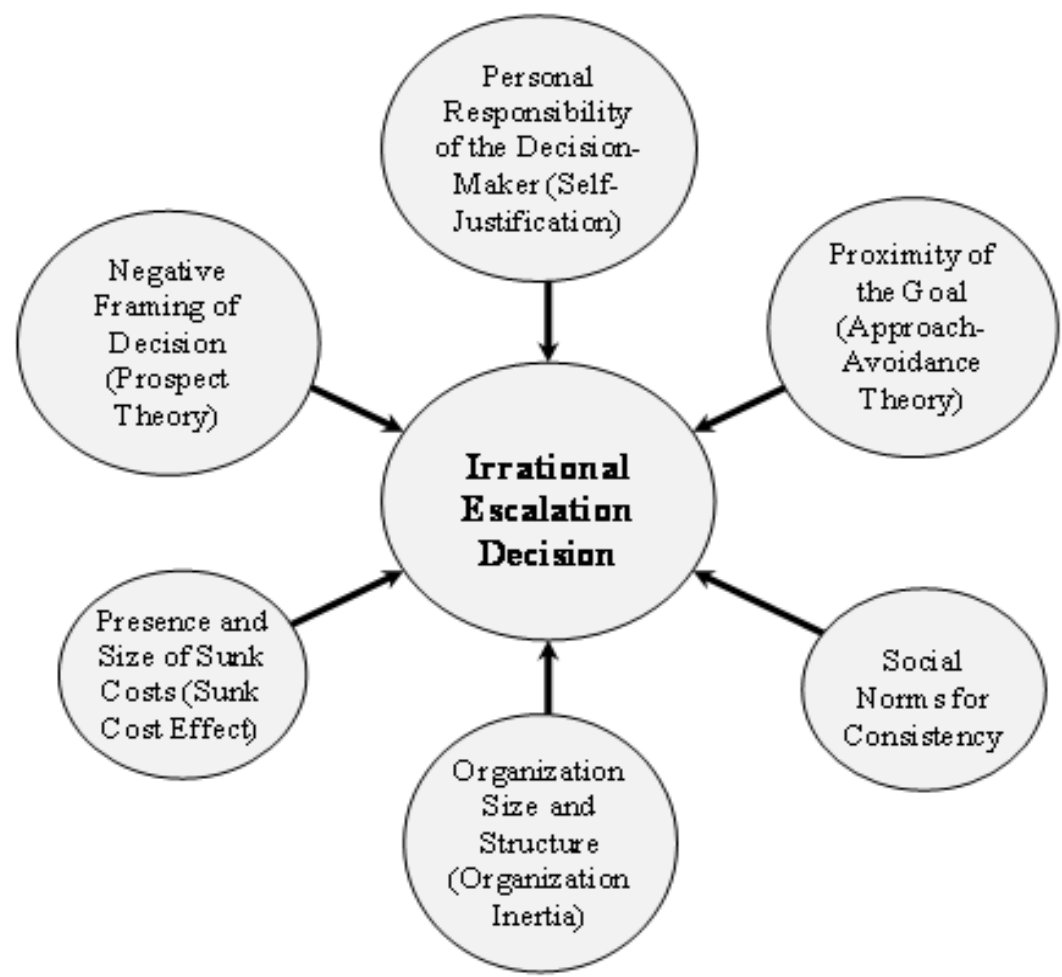

Fig. 2 Irrational escalation model

The specific de-escalation techniques to be used depend on the escalation determinant involved. For instance, if the irrational escalation decision is the outcome of self-justification, then a change in project management or rotation of duties can be recommended. An incoming manager that did not select the original project does not have the psychological commitment to the project. Keil and Robey (1999) identify management change as a 
de-escalation factor in the decisions of IS auditors. Ghosh (1997) shows that monitoring measures, such as regular progress reports, may reduce escalation. McNamara, et al (2002) present evidences that monitoring and changes in management are associated with de-escalation in banking managers' decisions on credit. In summary, management changes, governance procedures and monitoring may serve as useful instruments for de-escalation as they facilitate regular evaluation of a project and put a cost, either in monetary or career terms, on continuation of failing projects.

\section{Rational escalation}

An irrational escalation decision to continue a failing IS project is a true mistake for both the manager and the firm. However, not all escalation decisions are irrational, and some may be the outcome of a rational decision-making process by the manager. A model of rational escalation will be helpful in identifying the incentives of the managers and the firms for the continuation of projects that have received negative feedback.

\subsection{Agency theory}

The agency theory focuses on the conflict of interest between the agent (manager) and the principal (firm). Mahaney and Lederer (2003) present interview evidence that IS managers commonly encounter the agency conflict. The project continuation decision may be irrational for the firm, but rational for the manager as managers need to protect reputation. The manager in charge of the project is often the first to discover the likelihood of the project's success. Terminating a project that was originally selected or governed by the manager sends the signal that a mistake was made, and then the manager's reputation is damaged. If the future wages and career options of the manager depend on this reputation, there is an incentive to continue the project and conceal the negative information as described by Kanodia, et al (1989).

A related determinant of rational project continuation by the agent in this principal-agent framework is the nature of agent's incentives. If the agent is compensated based only on the project's performance, then there is an incentive to conceal negative information and delay termination of the project, as the compensation of the agent would be reduced by any action that reveals negative information. Such incentive is enhanced if the manager has the opportunity to leave the firm before the project is completed and the outcome is revealed. This provides the rationale for compensating managers based on their decision process, and not only on the outcome.

Escalation due to reputation protection in the agency model is rational for the manager, but value-reducing for the firm. The firm would prefer stopping failing projects as soon as the manager gets the negative information about the project's outcome. When the agency theory conflict of interest affects the incentives of the manager, the firm may even have an incentive to reward managers for revealing project failures in order to stop the cycle of escalation.

\subsection{Real option theory}

A different view of the escalation process is provided by the economic theories that examine project evaluation decisions and question whether the evaluation of the existing project and the available alternatives includes all costs and benefits of each option. As IS projects have an inherent high degree of uncertainty, the evaluation of available signals about the projects' likelihood of success is critical for optimal decision making. Failure to account for the full costs and benefits of each option, such as the value of real options embedded in a 
project and the value of investigating available technologies in the optimal sequence as dictated by the bandit theory may lead to premature termination of IS projects.

The traditional approach to project evaluation is the use of net present value (NPV) of a project's discounted cash flows. Dos Santos (1991), among others, criticizes the traditional NPV for its inability to include strategic aspects of IS projects into evaluation. These strategic aspects are captured by a real option when it offers the opportunity, but not the obligation, to make adjustments to the project in response to endogenous and exogenous events (Benaroch \& Kauffman, 1999). Full value of a project includes both the traditional NPV and the sum of the value of all real options. Ozogul, et al (2009) introduce a real options-based methodology which overcomes the limitations of traditional valuation methods, and enables decision-makers to value an IS investment incorporating multiple options. Wu, et al (2008) discuss the real option perspective on ERP implementation and point out that incorporating real options in the ERP evaluation process allow managers to improve their flexibility, take appropriate action to respond to environment shifts, and achieve a more successful ERP implementation.

Real option types encountered in IS projects are summarized by Tiwana, et al (2006). First, the Option to switch use refers to the opportunity to use the IS for an additional purpose from that for which it was originally intended. Second, the Option to change scale allows the scale of the developed system to be extended or contracted in the future. Third, the Option to stage investments allows the project to be funded incrementally, so that the project may be terminated when negative signals are revealed. Fourth, the Abandonment option involves the opportunity to discontinue the project prior to completion and redeploy remaining project resources without major negative effects on the firm. Fifth and final, the Growth option is associated with a project when an initial investment opens the door for potential additional investments, such as additional uses of the technology or additional markets for the developed IS solution.

These types of real options provide value to the firm that is not captured by basic NPV analysis. Taudes, et al (2000) report that real option value may represent a significant part of the full project value and make projects that have negative NPV worth continuing. For instance, the use of growth options is discussed in Taudes, et al (2000) with a case study of an European automaker that justified an investment in an enterprise resource planning system as a gateway to subsequent IS projects in a variety of areas including electronic purchasing and invoicing, workflow applications, e-commerce and document management.

\subsection{Bandit process theory}

Another model that focuses on the decision making process and complements the findings of the real option theory is the bandit process theory (Chulkov \& Desai, 2005). A bandit process is encountered in IS projects that involve choosing between two or more alternative systems configurations or technologies, when only one of the options may be investigated at a time. Furthermore, only one of the options is ultimately used by the firm. The solution to the bandit problem suggests that it is optimal to investigate the high-risk high-reward project first in the hope of "hitting the jackpot" early and ending the search.

Gittins (1989) as well as Banks and Sundaram (1992) outlined the solution techniques for the bandit-type decision problems. This term originates from studies of a gambler's choice between several slot machines (one-armed bandits). In terms of project selection, the bandit problem exists when the firm chooses between two or more alternative projects. The projects may be sampled sequentially, one at a time. The projects are of different types, providing either a high reward with a low probability of success or a low reward with a high probability of 
success. The type of the project is not known to the firm in advance, only the distribution of types is available. To learn the project's type, the firm must select it for at least one period. The problem is to identify which project should be realized first. If the project fails, the firm can then investigate the other option.

The optimal solution to the bandit problem is to investigate the high-risk high-reward project first. Since the reward of the project is unknown, and can only be learned by sampling the project, and the uncertainty regarding the high-risk high-reward project in the sense of the dispersion of rewards is greater, it is beneficial to resolve this uncertainty first. Formally, the optimal strategy involves the calculation of a reservation price (Weitzman, 1979) or index (Gittins, 1989) for each project. The optimal strategy is to choose the project with the highest reservation price (index). The search will stop when the highest obtained reward exceeds the reservation price for every remaining project. Weitzman (1979) demonstrates that as project rewards become more dispersed, the reservation price (index) increases. Therefore, it is optimal to try a risky project with a wider distribution of rewards before a safer project. When the safe option is selected first, the project has a lower maximum reward. Since only one system solution is ultimately used by the firm, this results in forfeiting the potential maximum reward of the risky project that is left unexplored. When the assumptions of the bandit theory are satisfied, it is optimal to select the high-risk option before the safer one even when the former has a lower probability of success.

De-escalation studies recommend governance mechanisms that prevent continuation of projects that have low likelihood of success. In contrast, the bandit theory demonstrates that in the case, when the firm sequentially evaluates competing technologies, it may be optimal to investigate the high-reward technology first, even if it has a low probability of success. Mechanisms that reduce escalation are in conflict with this implication of the bandit theory. In fact, firms that face the bandit problem will find in some periods that the optimal decision process of selecting high-risk projects may be associated with the termination of unsuccessful projects, and the firm will investigate the low-risk project in the following period. This is not indicative of an irrational escalation decision error and is only the effect of resolving uncertainty about the high-reward projects first.

\subsection{Rational escalation model}

Escalation is rational when it is justified by the value that the firm receives from investigating the project further. Keil and Mann (1997) report that up to forty percent of IS projects exhibit some degree of escalation. However, not all of these IS projects involve irrational errors in judgment. There are two types of rational escalation decisions. The real option theory and the bandit theory illustrate cases when project continuation is justified by the value of information and the value of flexibility that the firm receives from continuing the project. In this case, escalation is rational for both the manager and the firm. This escalation should not be stopped. In contrast, agency theory addresses the incentives of the manager that make escalation rational for the manager, but not the firm. Reputation protection by the managers may lead to continuation of failing projects, and de-escalation techniques should be utilized to prevent this type of escalation.

Fig. 3 summarizes the factors that are associated with rational escalation and highlights the cases that may provide value for the firm and make project termination premature. First, the real option theory suggests that future flexibility adds value to the project. The real option theory applies when there is uncertainty about the project, and continuing the project may yield new information or provide future growth opportunities for the firm. De-escalation techniques used by a firm must account for the value of real options embedded in the firm's projects. An observed negative NPV is not a sufficient signal of irrational escalation, as it does not represent the full value 
of the project to the firm. Aggressive use of benchmarks such as the NPV may limit innovation by terminating projects that provide future growth opportunities for the firm. Second, the bandit theory implies that the sequential nature of investigating alternative technological solutions yields value for exploring the high-risk high-reward projects before safer ones. The bandit problem exists when only one technology choice is implemented by the firm each period and only one is ultimately used. The optimal solution for the bandit problem involves investigating high-risk high-reward technologies fully before opting for a low-risk low-reward solution. De-escalation mechanisms that guide managers toward low-risk projects may not be in the best interest of the firm as they will cause high-reward projects to remain unexplored.

Not all cases of rational escalation are optimal for the firm. The agency theory implies that in cases when the managers are delegated with authority of selecting and overseeing a project, reputation concerns and compensation mechanism design may lead the manager to continue failing projects. This escalation is rational for the manager, but reduces value for the firm. De-escalation techniques such as rotation of duties, monitoring, and management change (Keil \& Robey, 1999) are recommended for preventing this type of rational escalation. If the project was selected by another decision maker, then there is less potential for reputation damage in stopping the escalated project. Governance procedures and monitoring may facilitate de-escalation in these situations by external evaluation of the project and by putting a cost on the continuation of failing projects.

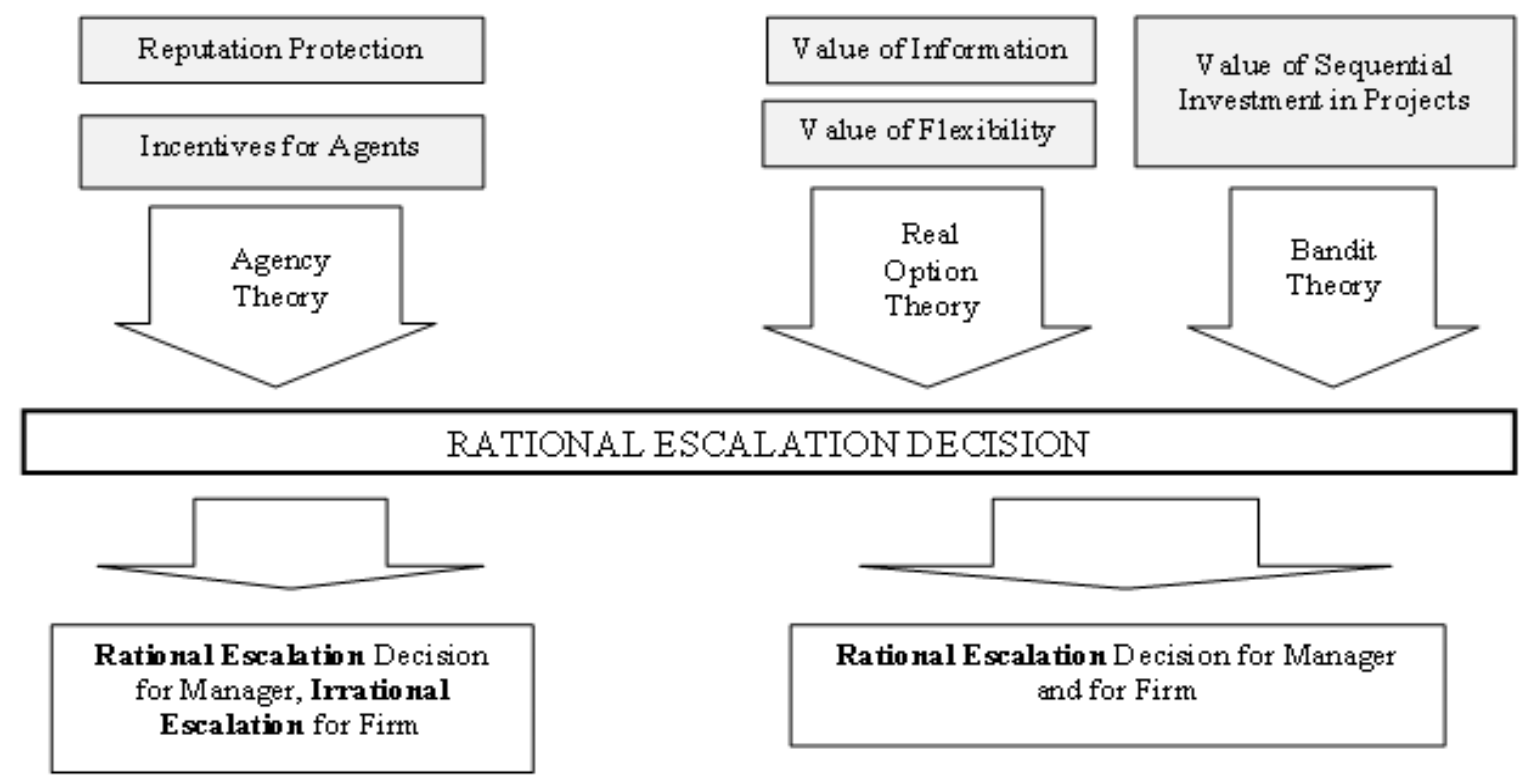

Fig. 3 Rational escalation model

\section{Conclusion}

Continuation of IS projects after receiving negative feedback is referred to as escalation of commitment. Such escalation is especially common in IS projects (Keil \& Mann, 1997; Keil, et al., 2000). This study summarizes the determinants of escalation into two groups that unite the factors leading to irrational escalation decisions and the factors leading to rational escalation decisions. A model of irrational escalation and a model of rational escalation are presented in order to discuss the factors that promote each type of project continuation 
decisions.

An analysis of factors in the irrational escalation model leads to a better understanding of the forces that contribute to irrational escalation, including the responsibility of the same manager for both the project selection and project continuation decisions that creates proneness to self-justification, the potential for negative framing of decision options due to large sunk costs, the large size of organization and the presence of organizational inertia. Understanding these irrational escalation factors helps address the problem of IS project waste. When the decision making process on an IS project encounters the determinants of irrational escalation, the utilization of de-escalation techniques is recommended, including a change in the management of the project, rotation of duties, and improved governance mechanisms such as monitoring and regular evaluations of the project's progress.

The rational escalation model highlights the fact that some escalation is rational and is in the best interest of both the manager and the firm. The real option theory and the bandit theory provide examples when project continuation is justified by the value of information and the value of flexibility that the firm receives from continuing the project. Understanding the nature of rational escalation allows the firm to promote project management practices that are in the best interest of the firm and avoid premature termination of projects.

Note, however, that not all rational escalation is optimal for the firm. Reputation protection and attempts to escape lower compensation due to project termination may lead a manager to continue a project after receiving negative feedback as long as there is informational asymmetry and the principal of the firm does not discover true project status immediately. The de-escalation techniques that may be used in agency situations are similar to the recommendations for irrational escalation, and include a change of the project's management or rotation of duties, and enhanced monitoring. Correct application of de-escalation techniques is helpful in improving system development success rate and the reduction of waste due to escalation errors.

\section{References:}

Abdel-Hamid, T. K.. (1988). Understanding the “90\% syndrome” in software project management: A simulation-based case study. Journal of Systems and Software, 8(4), 319-330.

Banks, J. \& Sundaram, R.. (1992). Denumerable-armed bandits. Econometrica, 60(5), 1071-1096.

Benaroch, M. \& Kauffman, R.. (1999). A case for using real options pricing analysis to evaluate information technology project investments. Information Systems Research, 10(1), 70-86.

Brockner, J.. (1992). The escalation of commitment to a failing course of action: Toward theoretical progress. Academy of Management Review,17(1), 39-61.

Brockner, J. \& Rubin, J.. (1985). Entrapment in escalating conflicts: A social psychological analysis. New York: Springer-Verlag.

Chulkov, D. \& Desai, M.. (2005). Information technology project failures: Applying the bandit problem to evaluate managerial decision making. Information Management and Computer Security, 13(2), 135-143.

Dos Santos, B.. (1991). Justifying investments in new information technologies. Journal of Management Information Systems, 7(4), 71-90.

Drummond, H.. (2005). What we never have, we never miss? Decision error and the risks of premature termination. Journal of Information Technology, 20, 170-176.

Garland, H., Sandefur, C. \& Rogers, A.. (1990). De-escalation of commitment in oil exploration: When sunk-costs and negative feedback coincide. Journal of Applied Psychology, 75, 721-727.

Ghosh, D.. (1997). De-escalation strategies: Some experimental evidence. Behavioral Research in Accounting, 9, 88-113.

Gittins, J.. (1989). Multi-armed bandit allocation indices. New York: Wiley.

Kahnemann, D. \& Tversky, A.. (1979). Prospect theory: Analysis of decisions under risk. Econometrica, 47, $263-291$.

Kanodia, C., Bushman, R. \& Dickhaut, J.. (1989). Escalation errors and the sunk cost effect: An explanation based on reputation and information asymmetries. Journal of Accounting Research, 27(1), 59-77. 
Keil, M. \& Mann, J.. (1997). The nature and extent of IT project escalation: Results from a survey of IS audit and control professionals. IS Audit Control Journal, 1, 40-48.

Keil, M., Mann, J. \& Rai, A.. (2000). Why software projects escalate: An empirical analysis and test of four theoretical models. Management Information Systems Quarterly, 24(4), 631-664.

Keil, M. \& Robey, D.. (1999). Turning around troubled software projects: An exploratory study of de-escalation of commitment to failing courses of action. Journal of Management Information Systems, 15(4), 63-87.

Kim, E. \& Park, Y.. (2007). Prediction of IS project escalation based on software development risk management. Journal of Information and Knowledge Management, 6(2), 153-163.

Mahaney, R. \& Lederer, A.. (2003). Information systems project management: An agency theory interpretation. Journal of Systems and Software, 68(1), 1-9.

McNamara, G., Moon, H. \& Bromiley, P.. (2002). Banking on commitment: Intended and unintended consequences of an organization's attempt to attenuate escalation of commitment. Academy of Management Journal, 45(2), 443-452.

Monteleagre, R. \& Keil, M.. (2000). De-escalating information technology projects: Lessons from the Denver international airport. Management Information Systems Quarterly, 24(3), 417-447.

Na, K., Li, X., Simpson, J. \& Kim, K.. (2004). Uncertainty profile and software project performance: A cross-national comparison. Journal of Systems and Software, 70(1-2), 155-163.

Ozogul, C. O., Karsak, E. E. \& Tolga, E.. (2009). A real options approach for evaluation and justification of a hospital information system. Journal of Systems and Software, 82(12), 2091-2102.

Rubin, J. \& Brockner, J.. (1975). Factors affecting entrapment in waiting situations: The Rosencrantz and Guildenstern effect. Journal of Personality and Social Psychology, 31, 1054-1063.

Staw, B.. (1976). Knee-deep in the big muddy: A study of escalating commitment to a chosen course of action. Organizational Behavior and Human Performance, 16, 27-44.

Staw, B.. (1981). The escalation of commitment to a course of action. Academy of Management Review, 6, 577-587.

Taudes, A., Feurstein, M. \& Mild, A.. (2000). Options analysis of software platform decisions: A case study. Management Information Systems Quarterly, 24(2), 227-243.

Tiwana, A., Keil, M. \& Fichman, R.. (2006). Information systems project continuation in escalation situations: A real option model. Decision Science, 37(3), 357-391.

Weitzman, M.. (1979). Optimal search for the best alternative. Econometrica, 47(3), 641-654.

Wu, L., Ong, C. \& Hsu, Y.. (2008). Active ERP implementation management: A real options perspective. Journal of Systems and Software, 81(6), 1039-1050.

Zmud, R. W.. (1980). Management of large software efforts. Management Information Systems Quarterly, 4, 45-55.

(Edited by Ruby and Chris) 